Review

\title{
OSBP-related protein 2 (ORP2): Unraveling its functions in cellular lipid/ carbohydrate metabolism, signaling and F-actin regulation
}

\author{
Vesa M. Olkkonen ${ }^{\mathrm{a}, \mathrm{b}, *}$, Annika Koponen ${ }^{\mathrm{a}, \mathrm{b}}$, Amita Arora ${ }^{\mathrm{a}, \mathrm{b}}$ \\ ${ }^{a}$ Minerva Foundation Institute for Medical Research, Biomedicum 2U, FI-00290, Helsinki, Finland \\ ${ }^{\mathrm{b}}$ Department of Anatomy, Faculty of Medicine, FI-00014, University of Helsinki, Finland
}

\section{A R T I C L E I N F O}

\section{Keywords:}

Akt

Actin cytoskeleton

Energy metabolism

Cholesterol transport

OSBPL2

Triglyceride

\begin{abstract}
A B S T R A C T
Oxysterol-binding protein (OSBP)-related proteins (ORPs) constitute a family of intracellular lipid-binding/ transport proteins (LTPs) in eukaryotes. They typically have a modular structure comprising a lipid-binding domain and membrane targeting determinants, being thus suited for function at membrane contact sites. Among the mammalian ORPs, ORP2/OSBPL2 is the only member that only exists as a 'short' variant lacking a membrane-targeting pleckstrin homology domain.

ORP2 is expressed ubiquitously and has been assigned a multitude of functions. Its OSBP-related domain binds cholesterol, oxysterols, and phosphoinositides, and its overexpression enhances cellular cholesterol efflux. Consistently, the latest observations suggest a function of ORP2 in cholesterol transport to the plasma membrane $(\mathrm{PM})$ in exchange for phosphatidylinositol 4,5-bisphosphate (PI4,5 $\left.\mathrm{P}_{2}\right)$, with significant impacts on the concentrations of PM cholesterol and PI4,5 $\mathrm{P}_{2}$. On the other hand, ORP2 localizes at the surface of cytoplasmic lipid droplets (LDs) and at endoplasmic-reticulum-LD contact sites, and its depletion modifies cellular triglyceride (TG) metabolism. Study in an adrenocortical cell line further suggested a function of ORP2 in the synthesis of steroid hormones. Our recent knock-out of ORP2 in human hepatoma cells revealed its function in hepatocellular PI3K/Akt signaling, glucose and triglyceride metabolism, as well as in actin cytoskeletal regulation, cell adhesion, migration and proliferation. ORP2 was shown to interact physically with F-actin regulators such as DIAPH1, ARHGAP12, SEPT9 and MLC12, as well as with IQGAP1 and the Cdc37-Hsp90 chaperone complex controlling the activity of Akt. Interestingly, mutations in OSBPL2 encoding ORP2 are associated with autosomal dominant non-syndromic hearing loss, and the protein was found to localize in cochlear hair cell stereocilia.

The functions assigned to ORP2 suggest that this protein, in concert with other LTPs, controls the subcellular distribution of cholesterol in various cell types and steroid hormone synthesis in adrenocortical cells. However, it also impacts cellular TG and carbohydrate metabolism and F-actin-dependent functions, revealing a bewildering spectrum of activities.
\end{abstract}

\section{Introduction}

ORP2 was initially discovered in 1999 in a screen for oxysterolbinding protein (OSBP) related expressed sequence tags [1]. The fulllength human cDNA was reported in 2001 [2-4] and the mouse orthologue in 2002 [5]. The ORP2 mRNA was observed ubiquitously in human [1] and mouse [5] tissues. ORP2 belongs, based on its amino acid sequence and gene structure, to ORP subfamily II, together with the closely related ORP1 [3]. ORPs share a characteristic $\beta$-barrel- resembling ligand-binding domain designated OSBP-related domain (ORD) in their carboxy-terminal region [3,6-8]. While all other mammalian OSBPL/Osbpl genes encode ORP proteins of the 'long' subtype, carrying an amino-terminal extension that contains a pleckstrin homology (PH) domain, ORP2 only exists as a 'short' subtype protein lacking a $\mathrm{pH}$ domain (Fig. 1; [2,3,5]). Another membrane targeting determinant present in most ORPs is called two phenylalanines in an acidic tract (FFAT) which mediates binding of the protein to the integral type 2 membrane proteins of the endoplasmic reticulum (ER),

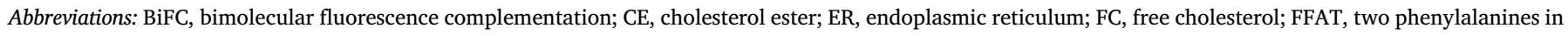

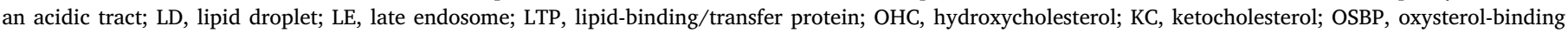

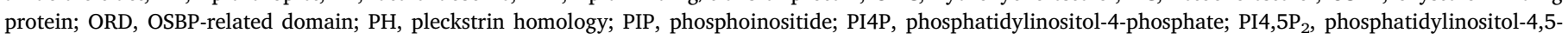
bisphosphate; $\mathrm{PI} 3,4,5 \mathrm{P}_{3}$, phosphatidylinositol-3,4,5-trisphosphate; PM, , plasma membrane; SPR, surface plasmon resonance; TG, triglyceride

* Corresponding author at: Minerva Foundation Institute for Medical Research, Biomedicum 2U, Tukholmankatu 8, FI-00290, Helsinki, Finland.

E-mail address: vesa.olkkonen@helsinki.fi (V.M. Olkkonen).
} 


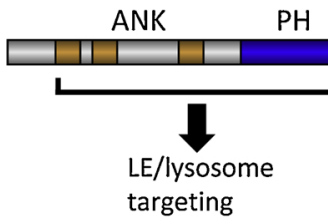

100 aa

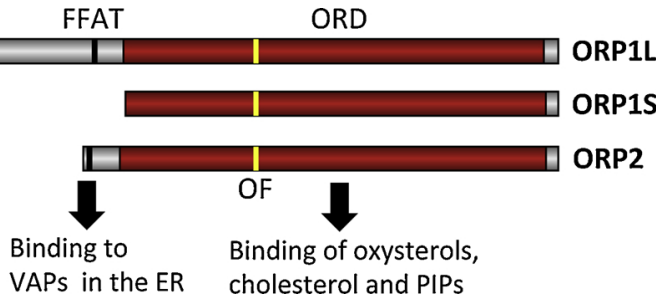

Fig. 1. Alignment of ORP2 protein with the closely related members in subfamily II, ORP1L and ORP1S. ANK, ankyrin repeats; $\mathrm{PH}$, pleckstrin homology domain; FFAT, two phenylalanines in an acidic tract; ORD, OSBP-related (ligand-binding) domain; OF, OSBP fingerprint sequence (EQVSHHPP), a conserved sequence motif with a crucial role in phosphoinositide binding. LE, late endosome; VAP, VAMP-associated protein; ER, endoplasmic reticulum; PIP, phosphoinositide.
VAMP-associated proteins A and B (VAPA,VAPB [9];). For two ORPs (ORP5 and ORP8) this functional motif is replaced by an ER-targeting trans-membrane segment [10,11].

The presence of the ER-targeting motifs and $\mathrm{pH}$ domains or a transmembrane segment confers ORPs the capacity to associate with two distinct membrane organelles at membrane contact sites (MCSs), and the current paradigm postulates that ORPs execute lipid transport and signaling functions at such intimate, direct organelle contacts [12-14]. The lipid transport function of ORPs involves a 'countercurrent' mechanism, in which a substrate lipid (thus far evidence exists for cholesterol or phosphatidylserine) is transferred in exchange for phosphatidylinositol-4-phosphate (PI4P [15-20];) or another polyphosphoinositide such as $\mathrm{PI}_{4}, 5 \mathrm{P}_{2}$ [21]. A crucial part of the transport cycle is the hydrolysis of the countertransported PI4P by the ER phosphoinositide phosphohydrolase Sac1 [22]. ORP2 does carry close to its amino-terminus a FFAT motif and thus binds to VAP proteins at the ER [23]. However, it lacks the $\mathrm{pH}$ domain and associated membrane targeting function. In this focused review we summarize the current knowledge on the function of ORP2.

\section{Tissue expression pattern and subcellular localization of ORP2}

The early reports on the identification of ORP/OSBPL sequences demonstrated that the ORP2 mRNA is detectable in all human and mouse tissues [1-3,5,24]. In western analysis of mouse tissues also the protein was observed in all tissues, with the strongest signal detected in the brain [24]. Two protein variants, with the apparent molecular masses of 51 and $56 \mathrm{kDa}$ in SDS-PAGE were observed, with tissuespecific differences in their abundance. The identity of these protein variants has not been determined.

Cell fractionation by Laitinen et al. [24] suggested that a major portion of the endogenous ORP2 in Chinese hamster ovary (CHO) cells is cytosolic; however, part of the protein was found in the membrane fraction. Early overexpression studies in $\mathrm{CHO}$ cells indicated localization of ORP2 at the Golgi complex, consistent with a Golgi vesicle transport defect observed in CHO cells overexpressing this protein $[4,24]$. However, later work revealed prominent localization of ORP2 on the surface of cytoplasmic lipid droplets (LDs) in A4312, HeLa, HepG2 and COS-7 cells, with no detectable Golgi targeting [25]. Of interest, incubation of cells with the high affinity ligand of ORP2, 22(R)-hydroxycholesterol (OHC), resulted in a different, diffuse and cell cortex/plasma membrane (PM) localization of the protein [25]. A sitespecific mutant deficient in oxysterol binding, ORP2 I249 W [26], showed enhanced association with LDs and could not be detached therefrom with 22(R)OHC, suggesting that oxysterol binding controls the conformation of ORP2 and this, in turn, plays a crucial role in its targeting to LDs [25].

Several years later, Weber-Boyvat et al. [23] investigated in $\mathrm{HuH7}$ hepatoma cells the subcellular localization of complexes of ORP2 with its ER anchor, VAPA, by using the bimolecular fluorescence complementation (BiFC) technique. They detected ORP2-VAPA complexes at ER-LD contacts. Moreover, siRNA-mediated knock-down of ORP2 and VAPs enhanced the turn-over of cellular triglycerides. These observations suggested that a major function of ORP2 could involve the ER-LD interfaces and the TG metabolic processes occurring therein. However, the study of Kentala et al. [27] revealed that the loss of ORP2 did not reduce ER-LD contacts, but under basal culture conditions rather increased them. However, this work indicated that the dynamic expansion of the ER-LD MCSs upon fatty acid loading may be disturbed in the ORP2 KO cells. Similar to ORP2 expressed alone, ORP2-VAPA BiFC complexes were upon 22(R)OHC treatment redistributed from LDassociated ER structures to elongated membraneous-appearing elements and occasionally to the cell cortex/PM [28]. Cell cortex localization of ORP2 was also observed in human umbilical vein endothelial cells (HUVECs) that seldom have large LDs. In these cells, the protein was frequently found co-localizing with the actin regulator cortactin in lamellipodia [27].

If cells have large LDs or are subjected to fatty acid loading to increase LD size, ORP2 associates prominently with LDs (Fig. 2A). However, many cultured cell lines display under standard growth conditions only very small or undetectable LDs. Under these conditions the cytosolic and other non-LD aspects of ORP2 localization, such as the cell cortex/PM and lamellipodia (Fig. 2B), predominate. The recent study of Koponen et al. [29] revealed a previously undiscovered localization of the protein on late endosomes (LE) labeled with anti-Lamp1 or internalized fluorescent dextran (Fig. 2C).

To summarize, exogenously expressed ORP2 targets LDs or ER-LD contacts, but in the absence of large LDs, it remains largely cytosolic or and/or targets the cell cortex/PM and LEs. When co-expressed with VAPA, ORP2 is seen attached to the ER and prominently at ER-LD contacts. A major problem is that, with the antibodies we have generated or those available commercially it has been difficult to reliably detect the endogenous cellular ORP2. Some efforts have, however, been made. Our study [25] suggested that some endogenous ORP2 may be detectable on the LDs of fatty acid loaded A431 cells. Thoenes et al. [30] reported detection of endogenous ORP2 in the stereocilia of mouse cochlear inner and outer hair cells. Moreover, Escajadillo et al. [31] reported detection of endogenous ORP2 within the nucleus of adrenocortical H295R cells, consistent with their data suggesting its association with the transcription factor liver $\mathrm{X}$ receptor (LXR). However, new antibodies of better quality and independent confirmations in different cell types, validated by ORP2 knock-down or knock-out, are required before firm statements can be made on the subcellular localization of endogenous ORP2.

\section{Ligands of ORP2}

A number of reports have addressed the lipid ligands of ORP2 by employing charcoal-dextran, pull-down, lipid-protein overlay assays, extraction of lipids from vesicles, or surface plasmon resonance (SPR) (Table 1). Initially, the group of N. Ridgway investigated the lipidous ligands of ORP2 by using a lipid-protein overlay assay. The results suggested binding of ORP2 to the acidic lipids phosphatidic acid (PA), phosphatidylinositol-3-phosphate (PI3P) and cardiolipin [4]. These results need to be interpreted with caution, since lipids spotted on nitrocellulose surface are presented to proteins in a rather non-physiologic manner. Our group next documented, by using a charcoal-dextran assay, binding of the oxysterol $25 \mathrm{OHC}$ to ORP2 produced in E.coli as a GST fusion, at a $3.9 \times 10^{-6} \mathrm{M} \mathrm{K}_{\mathrm{d}}$ [26]. In a follow-up study [25] we measured affinities of ORP2 for 22(R)OHC $\left(1.4 \times 10^{-8} \mathrm{M}\right)$ and 7-ketocholesterol $\left(7 \mathrm{KC} ; 1.6 \times 10^{-7} \mathrm{M}\right)$ and presented evidence that the protein also binds cholesterol, based on a pull-down assay in which 

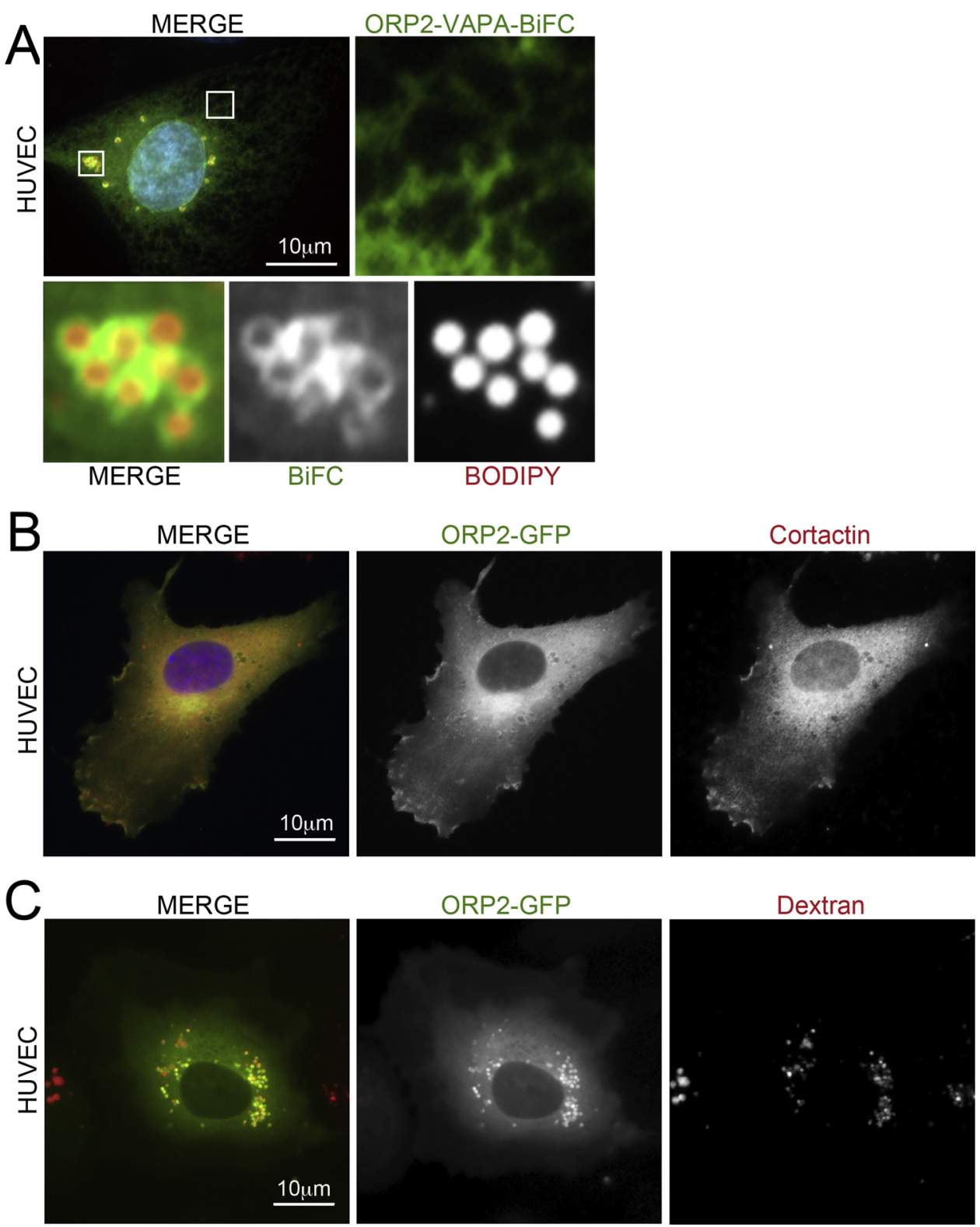

Fig. 2. Fluorescence microscopy images depicting the various subcellular localizations of ORP2. A. Biomolecular fluorescence complementation (BiFC) analysis of ORP2-VAPA complexes in human umbilical vein endothelial cells (HUVECs) transfected for $24 \mathrm{~h}$ by electroporation (Nucleofector ${ }^{\mathrm{TM}}$, Lonza Bioscience). Note the reticular ER pattern and intense staining at ER domains encircling lipid droplets (LDs) stained with BODIPY-C 12 (Molecular Probes/Thermo Scientific). B. Localization of ORP2-GFP at the cell cortex, where it co-localizes with the lamellipodial marker cortactin (stained with monoclonal antibody 4F11; Upstate Biotechnology). C. Localization of ORP2-GFP in late endosomes/lysosomes identified by using internalized Alexa Fluor ${ }^{\mathrm{TM}} 647$ dextran (Molecular Probes/Thermo Scientific).
ORP2 extracted cholesterol from methyl- $\beta$-cyclodextrin. Our latest work confirmed the cholesterol binding, by employing a pull-down assay in which radiolabeled cholesterol was solubilized with the zwitterionic detergent CHAPS. However, the binding did not reach saturation, so we were unable to determine a $K_{d}$ for this interaction [29].

In addition to a PIP-binding $\mathrm{pH}$ domain, several ORPs have the capacity to accommodate and extract PI4P and other PIPs by their ORD domain [15-17,21]. In 2005 we showed that ORP2 is capable of pulling down phosphatidylcholine (PC) vesicles with $2 \mathrm{~mol} \%$ (unlabeled) multiply phosphorylated PIPs, the pull-down being most efficient with $\mathrm{PI} 3,4,5 \mathrm{P}_{3}$ [32]. This suggested an interaction of ORP2 with the head groups of long-chain PIPs in vitro on vesicle surfaces, since extraction of a PIP by the ORD would not result in pull-down of the vesicles labeled with $\left[{ }^{14} \mathrm{C}\right] \mathrm{PC}$. In the latest study we assessed the binding of soluble

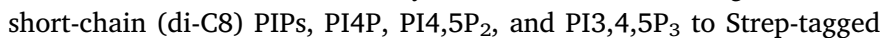
ORP2 immobilized on SPR chips. The signal obtained in this assay may represent binding of the PIPs within the ORD pocket, but also interaction of the PIPs with the surface of ORP2 could contribute to the signal. ORP2 bound PI4,5P 2 at $52 \mu \mathrm{M}, \mathrm{PI} 3,4,5 \mathrm{P}_{3}$ at $76 \mu \mathrm{M}$, and PI4P at a $305 \mu \mathrm{M} \mathrm{K} \mathrm{d}_{\mathrm{d}}$ [29]. However, the data need to be considered with some caution, since the folding status of ORP2 fusion proteins produced in $E$. coli has not been assessed in detail. Importantly, Wang et al. [33] recently solved the high-resolution structure of the ORP2 ORD with bound $\mathrm{PI} 4,5 \mathrm{P}_{2}$ and reported that the protein binds this PIP as a tetramer. They also demonstrated binding of the other six PIPs by employing an in vitro assay in which the ORD extracted the PIPs from vesicles (Table 1). The above reports suggest that the ORP2 ORD may show higher affinity for multiply phosphorylated PIPs than for PI4P. Published data suggests that $\mathrm{PI} 4,5 \mathrm{P}_{2}$ and possibly $\mathrm{PI} 3,4,5 \mathrm{P}_{3}$ may be the most important PIP ligands of ORP2 at the PM [29,32,33], and we hypothesize that it could bind $\mathrm{PI} 3,5 \mathrm{P}_{2}$ on LE/lysosomes, since ORP2 binds this late endocytic pathway membrane component quite efficiently on vesicle surfaces in vitro [32].

\section{Roles of ORP2 in cellular sterol and steroid hormone metabolism}

Overexpression of ORP2 in CHO or HeLa cells was initially found to enhance the efflux of cellular radioisotope-labeled cholesterol to all acceptors, including human serum, apoA-I, and small unilamellar phosphatidylcholine vesicles [24,32], as well as the appearance of metabolically labeled newly synthesized cholesterol at the cell surface 
Table 1

Lipidous ligands reported to bind to ORP2.

\begin{tabular}{|c|c|c|c|}
\hline Ligand & Assay method & $\mathrm{K}_{\mathrm{d}}$ & Reference \\
\hline Phosphatidic acid & Lipid-protein overlay & ND* & [4] \\
\hline Cardiolipin & Lipid-protein overlay & ND & [4] \\
\hline \multirow[t]{2}{*}{ PI3P ${ }^{\#}$} & $\begin{array}{l}\text { Lipid-protein } \\
\text { overlay }\end{array}$ & ND & [4] \\
\hline & $\begin{array}{l}\text { Extraction from } \\
\text { vesicles }\end{array}$ & ND & [33] \\
\hline \multirow[t]{2}{*}{ PI4P } & $\mathrm{SPR}^{\S}$ & $305 \times 10^{-6} \mathrm{M}$ & [29] \\
\hline & Extraction from vesicles & ND & [33] \\
\hline PI5P & Extraction from vesicles & ND & [33] \\
\hline \multirow[t]{2}{*}{$\mathrm{PI} 3,4 \mathrm{P}_{2}$} & Vesicle pull-down & ND & [32] \\
\hline & Extraction from vesicles & ND & [33] \\
\hline \multirow[t]{2}{*}{$\mathrm{PI} 3,5 \mathrm{P}_{2}$} & Vesicle pull-down & ND & [32] \\
\hline & Extraction from vesicles & ND & [33] \\
\hline \multirow[t]{5}{*}{$\mathrm{PI} 4,5 \mathrm{P}_{2}$} & Vesicle pull-down & ND & [32] \\
\hline & SPR & $52 \times 10^{-6} \mathrm{M}$ & [29] \\
\hline & $\begin{array}{l}\text { X-ray crystallogr. Extraction } \\
\text { from vesicles }\end{array}$ & & \\
\hline & & ND & [33] \\
\hline & & ND & [33] \\
\hline \multirow[t]{3}{*}{$\mathrm{PI} 3,4,5 \mathrm{P}_{3}$} & Vesicle pull-down & ND & [32] \\
\hline & SPR & $76 \times 10^{-6} \mathrm{M}$ & [29] \\
\hline & Extraction from vesicles & ND & [33] \\
\hline $22(\mathrm{R}) \mathrm{OHC}^{\epsilon}$ & Charcoal-dextran & $1.4 \times 10^{-8} \mathrm{M}$ & [25] \\
\hline $7 \mathrm{KC}^{\&}$ & Charcoal-dextran & $1.6 \times 10^{-7} \mathrm{M}$ & [25] \\
\hline \multirow[t]{2}{*}{$25 \mathrm{OHC}$} & Charcoal-dextran & $\mathrm{x} 10^{-6} \mathrm{M}$ & [26] \\
\hline & Photo-cross-linking & ND & [26] \\
\hline Cholesterol & Pull-down & ND & {$[25,29]$} \\
\hline Dehydroergosterol & Extraction from vesicles & ND & [33] \\
\hline
\end{tabular}

\footnotetext{
* Not determined.

\# Phosphatidylinositol-3-phosphate.

$\S$ Surface plasmon resonance.

$€$ Hydroxycholesterol.

\& Ketocholesterol.
}

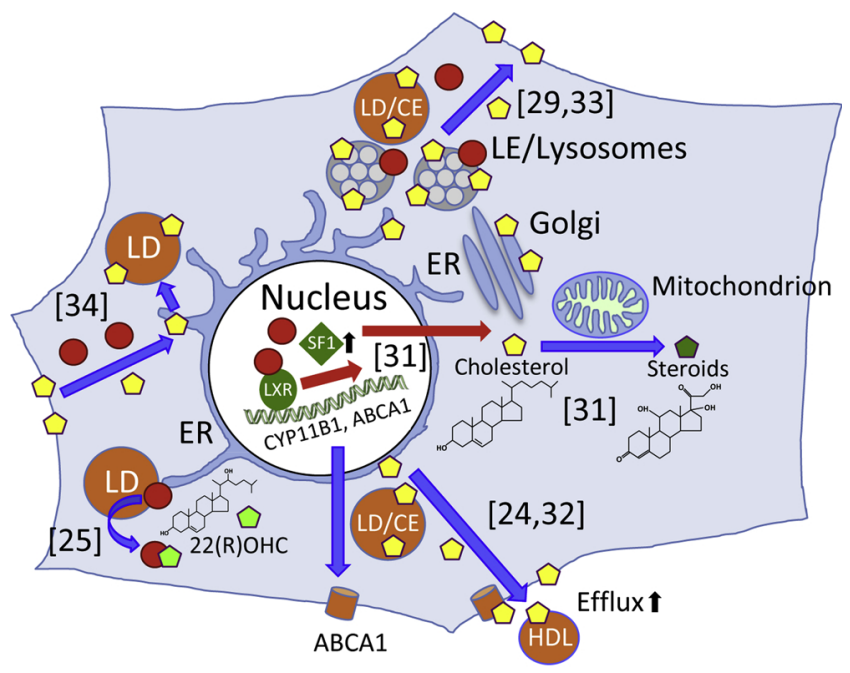

\section{ORP2 Cholesterol $\triangle$}

Fig. 3. A schematic image depicting the reported functions of ORP2 in cellular cholesterol and steroid metabolism. The references from which each piece of information originates are indicated in brackets. Published data suggest roles of ORP2 in cholesterol transport from late endosomes (LE)/lysosomes and possibly other compartments to the plasma membrane [29,33], in transcriptional regulation of sterol metabolism and steroidogenesis [31], in cholesterol efflux from cells [24,32], and cholesterol transport from the plasma membrane to the ER and lipid droplets (LD) [34]. ORP2 is detached from LDs by the high-affinity ligand 22(R)-hydroxycholesterol [22(R)OHC] [25]. LXR, liver X receptor; SF1, steroidogenic factor 1; ER, endoplasmic reticulum; CE, cholesterol ester; CYP11B1, cytochrome P450 family 11 subfamily B member 1; ABCA1, ATPbinding cassette transporter A1; HDL, high-density lipoprotein. as detected by employing methyl- $\beta$-cyclodextrin [32](Fig. 3). These observations suggested that abundant ORP2 in the cytosol of the stably transfected cells facilitated the intracellular transport of cholesterol, resulting in its rapid appearance at the cell surface measured by employing the efflux process or by a wash of cell surfaces with methyl- $\beta$ cyclodextrin. The new hallmark study by the group of H. Yang [33] demonstrated the capacity of ORP2 to transport cholesterol to the PM in exchange for $\mathrm{PI} 4,5 \mathrm{P}_{2}$ : Its knock-out in HEK293 cells reduced the cholesterol and increased the PI4, $5 \mathrm{P}_{2}$ content of the $\mathrm{PM}$, as well as conferred the cells elevated resistance to the cholesterol-binding drug amphotericin B. Further evidence for a direct sterol/PI4,5P 2 exchange or 'countercurrent' transfer activity of ORP2 was produced by in vitro vesicle-to-vesicle lipid transfer assays. Jansen et al. [34] studied the transport of cholesterol from the PM to lipid droplets in HeLa cells. In an overexpression screen they identified ORP2 and another related 'short' ORP, ORP1S, as gene products whose overexpression speeded up the movement of PM cholesterol to LDs. In more detailed analyses the effect was localized to the initial PM to ER transport step (Fig. 3). To determine whether also depletion of these ORPs would impact the cholesterol transport process, siRNA-mediated knock-down was carried out. Here, the double knock-down of ORP2 and ORP1S resulted in a modest but significant reduction of cholesterol transfer to the ER and LDs, supporting the involvement of the endogenous cellular proteins in PM to ER cholesterol trafficking. Consistent with the observations of Wang et al. [33], Koponen et al. [29] observed in a recent study carried out with HeLa cells that knock-down of ORP2 or overexpression of a mutant defective in PIP binding resulted in the accumulation of cholesterol probes (a derivative of Clostridium perfringens toxin, $\mathrm{D} 4 \mathrm{H}$, or filipin) in late endosomes, while overexpression of wild-type ORP2 shifted the D4H probe to the PM. These observations support the idea that ORP2 plays an important role in intracellular cholesterol transport to the PM - from endosomes and possibly other subcellular compartments (Fig. 3). This would be consistent with the enhancement of cholesterol efflux upon ORP2 overexpression [24,32], but somewhat at odds with the data of Jansen et al. [34]. However, we find it possible that ORP2 may be involved in several routes of cholesterol transport.

Kentala et al. [27,35] generated HuH7 hepatoma cells entirely devoid of ORP2 by using the CRISPR-Cas9 technology. Analysis of the lipidome of these cells revealed no change in free cholesterol (FC), cholesterol ester (CE) or oxysterol (25OHC, 27OHC, $7 \alpha \mathrm{OHC}, 7 \beta \mathrm{OHC}$, 7KC) content [27]. Even challenge of the cells by sterol depletion by employing lipoprotein-deficient serum plus statin treatment, or by LDL loading, did not change the situation, suggesting that a chronic lack of ORP2 did not distort cholesterol homeostatic regulation in the hepatoma cells. However, next generation RNA sequencing of the cells revealed significant alterations in the mRNA expression of 11 key cholesterol homeostatic genes, including HMGCR and SOAT1, indicating that the depletion of ORP2 had disturbed the sterol homeostasis, but the effects had been compensated for by adaptation via altered gene expression [27].

The results of Escajadillo et al. [31] in the adrenocortical H295R cell line subjected to stable shRNA-mediated knock-down of ORP2 revealed 2.9-fold and 1.9-fold increases of cellular FC and 25OHC, respectively, as well as significant reductions of $22 \mathrm{OHC}$ and $7 \mathrm{KC}$. These changes coincided with significant reductions in multiple steroid metabolites, including progesterone, 11-deoxycortisol and cortisol, but increased concentrations of androgens and estrogens. Moreover, knock-down of ORP2 suppressed the expression of several proteins required for cortisol biosynthesis and of the steroidogenic transcription factor SF1. The authors presented evidence that ORP2 may interact with the nuclear receptor liver X receptor (LXR), and both proteins bind to the promoter of CYP11B1 in response to cAMP signaling. CYP11B1 encodes steroid $11 \beta$ hydroxylase, a key enzyme converting 11-deoxycortisol to cortisol in the adrenal cortex. However, also a function of ORP2 in the transport of cholesterol, a substrate for the synthesis of steroid hormones, could obviously affect steroidogenesis. The observations are consistent with 
an earlier finding by the same group identifying ORP2 as an interaction partner of the formin family protein diaphanous homologue 1 (DIAPH1), a Rho GTPase effector which regulates cortisol production by controlling the rate of mitochondrial movement [36]. Of note, DIAPH1 regulates cytoskeletal dynamics by interacting with actin, microtubules and other cytoskeletal-associated regulatory proteins ([37]; see section 7). The above observations suggest that ORP2 executes in steroidogenic cells specific functions in the synthesis of steroid hormones, transcriptional regulation apparently playing an important role therein (Fig. 3).

\section{Impacts of ORP2 manipulations on triglyceride metabolism}

Discovery of ORP2's affinity for LD surfaces [25] and a reduction of TGs observed in CHO cells stably overexpressing ORP2 [38] suggested that the protein may execute a function in cellular neutral lipid metabolism. We therefore analyzed the impacts of ORP2 knock-down in A431 cells on LD morphology and biochemically on the turn-over of cellular TGs. Upon ORP2 knock-down, the consumption of LDs in response to lipid starvation was slowed down in morphological analyses; Consistently, biochemical analyses revealed that the break-down of radioisotope-labeled TGs was slowed down in the knock-down cells [25]. These observations suggested that ORP2, presumably at the LD surface, may facilitate the consumption of stored TGs upon fatty acid depletion. Weber-Boyvat et al. [23] discovered in HuH7 hepatoma cells that knock-down of ORP2 together with its ER anchors VAPA and -B, speeded up the consumption of radioisotope-labeled TGs upon lipid depletion. Knock-down of ORP2 alone had in this case no significant effect on the TGs. The results appeared contradictory to those of Hynynen et al. [25], perhaps due to a different cell model employed, but nevertheless supported a functional role of ORP2 as a regulator of TG metabolism.

The complete knock-out of ORP2 in the HuH7 hepatoma cell model [27] revealed as the only significant lipidome change, reduced TG storage, and consistently, reduced synthesis of TGs in pulse labeling experiments. RNA sequencing showed a reduced expression of the lipogenic transcription factor SREBP-1 and a large number of its established target genes such as FASN and $S C D$, suggesting that the loss of ORP2 may result in transcriptional down-regulation of the hepatocellular lipogenesis. A possible underlying mechanism was proposed: We found defective activity of the Ser/Thr kinase Akt, a key regulator of cell metabolism and viability, in the ORP2 knock-out cells. Since Akt has been documented to control the activity of SREBP-1 [39-41], defective Akt signaling could provide a plausible explanation for the reduction of SREBP-1 dependent transcription and lipogenesis (Fig. 4). To summarize, a number of evidences based on acute knock-down or complete knock-out of ORP2 in different cell types support a functional role of the protein as a regulator of cellular TG metabolism, in addition to a function in cholesterol transport.

\section{Function of ORP2 in the carbohydrate metabolism of hepatoma cells}

Having consistently observed impacts of ORP2 knock-down/knockout on TG metabolism, we wanted to investigate whether these impacts actually extend wider in the cellular energy metabolism, including that of carbohydrates. Analyses of the ORP2 knock-out HuH7 hepatoma cells revealed significant defects in glucose uptake, glycogen synthesis and glycolysis [27] (Fig. 4). Since glucose metabolism is robustly regulated by insulin via the PI3K/Akt signaling pathway, we assessed the activating Ser473 phosphorylation of Akt and the Ser9 phosphorylation of Akt's target glycogen synthase kinase $3 \beta$ (GSK-3ß), a central regulator of glycogen synthesis [42]. We observed a substantial reduction of both phosphorylations, suggesting that the loss of ORP2 indeed results in defective Akt signaling (Fig. 4). The mechanisms through which ORP2 affects the activity of Akt require further study.

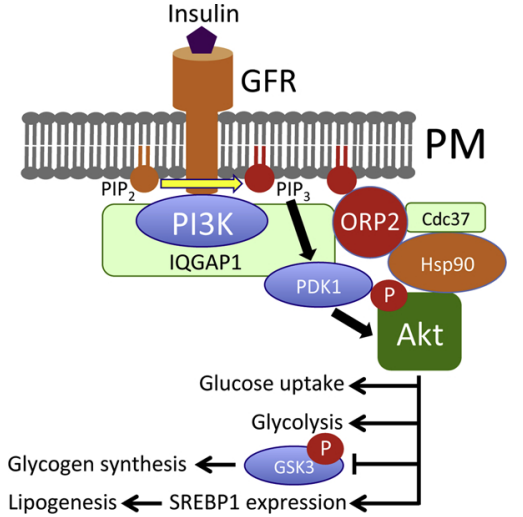

Fig. 4. A schematic model on the function of ORP2 in PI3K/Akt signaling and cellular energy metabolism. The Akt signaling pathway is stimulated by growth factors (such as insulin), which bind to plasma membrane (PM) growth factor receptors (GFR) resulting in the activation of phosphoinositide-3-kinase (PI3K). PI3K phosphorylates $\mathrm{PIP}_{2}$ to $\mathrm{PIP}_{3}$, which triggers the recruitment of phosphoinositide-dependent kinase 1 (PDK1) and Akt to the PM. ORP2 interacts with IQGAP1 which scaffolds the phosphoinositide kinases at the PM. Akt interacts at the PM with its effector complex Hsp90-Cdc37-ORP2 and is phosphorylated by PDK1. Active Akt regulates a number of downstream target molecules (including glycogen synthase kinase 3, GSK3), resulting in increased uptake of glucose, glycogen synthesis, glycolysis, and lipogenesis via sterol regulatory element binding protein-1 (SREBP-1).

However, we managed to find some clues of this: (i) ORP2 was found to physically interact with IQGAP1 [35], a protein acting as a scaffold for phosphoinositide kinases [43] in the cascade that eventually produces $\mathrm{PI}, 4,5 \mathrm{P}_{3}$, a key messenger in the PI3K/Akt signaling pathway and (ii) ORP2 was found to physically interact with Cdc37 and Hsp90 [27], which form a chaperone complex required for Akt activity [44]. The above results suggested that ORP2 not only regulates the cellular TG metabolism, but has more global impacts on energy metabolism. This function could be in part mediated by ORP2's involvement in interorganelle lipid transport, potentially resulting in altered lipid domain organization with secondary effects on signaling. However, we also identified protein-protein interactions that most likely are of importance. Of note, defective Akt signaling upon ORP2 depletion was not only observed in the cancerous hepatoma cell line, but also in primary human umbilical vein endothelial cells [27]. It will be extremely interesting to study insulin signaling, lipid and carbohydrate metabolism in vivo in Osbpl2 knock-out mice.

\section{ORP2 in cell F-actin regulation, adhesion, migration and proliferation}

The RNA sequencing of ORP2 knock-out hepatoma cells by Kentala et al. [35] revealed significant changes in the expression of genes in the functional categories 'Cellular movement', 'Cell-cell signalling and interaction', 'Cellular development', Cellular function and maintenance', 'Cellular growth and proliferation', 'Cell morphology', and 'Cell Death and Survival'. A number of pathways in these categories involved the actin cytoskeleton, cell migration, adhesion or proliferation. Moreover, analysis of the ORP2 protein interactome in HuH7 cells uncovered $>100$ putative new partners, pathway analysis of which revealed 'RhoA signalling' as the most significant ORP2-associated pathway. Interactions of ORP2 with the actin regulatory components SEPT9, MLC12, ARHGAP12 and IQGAP1 were validated by independent assays. Consistently, loss of ORP2 resulted in abnormal F-actin morphology characterized by impaired capacity to form lamellipodia, a migration defect and reduced rates of adhesion to substratum and proliferation. The results point at a novel function of ORP2 as a regulator of the actin cytoskeleton, with impacts on hepatocellular migration, adhesion, and proliferation. However, one has to bear in mind 
that the above data is based on work with cancerous hepatic cells. It is therefore important to carry out similar analyses in untransformed primary hepatocytes in the future.

\section{Mutations in OSBPL2 are associated with hereditary non- syndromic hearing loss}

Two studies reported in 2015 that human mutations in OSBPL2 associate with autosomal dominant non-syndromic hearing loss (ADNSHL) [30,45]. The syndrome is genetically heterogeneous: 65 loci and more than 30 causative genes have been designated. Thoenes et al. [30] carried out genome-wide linkage analysis and whole exome sequencing, identifying a co-segregating heterozygous frameshift mutation in OSBPL2, which truncates the encoded protein. Immunohistochemistry in mouse cochlea by using a commercial ORP2 antibody suggested the presence of ORP2 protein in the inner and outer hair cell stereocilia, structures which strongly depend on F-actin [46]. Similar observations were reported in a large Chinese family by Xing et al. [45], who also found a frameshift mutation in OSBPL2 co-segregating with the ADNSHL phenotype. Moreover, they found another missense mutation (c.583C > A transversion; p.Leu195Met) in the gene in a sporadic case of the disease. Although the causality of these mutations is not $100 \%$ certain, they make OSBPL 2 an obvious candidate gene for the disease, especially since also mutations in DIAPH1, an interaction partner of ORP2 [36], are reported to cause hearing loss [47]. We find it likely that the association of OSBPL2 mutations with hereditary hearing loss reflects a function of the protein in actin cytoskeletal regulation (see Section 7). Whether the functional cross-talk of ORP2 with the actin cytoskeleton involves sterol binding or transport by the protein is largely unclear; However, the capacity of ORP2 reintroduced into knock-out HuH7 cells to alter cell surface and F-actin morphology and to replenish migration in a wound healing assay depended on the ability of ORP2 to bind phosphoinositides [35], suggesting that the F-actin associated function of ORP2 involves the binding of PIPs by the protein.

\section{Conclusions and future perspectives}

Recent studies employing knock-down or knock-out of ORP2 in different cell types have suggested a bewildering multitude of functions for this lipid-binding protein: Cholesterol and PIP transport, steroid hormone synthesis, TG metabolism, F-actin regulation, cell adhesion, migration and proliferation. Although ORP2 lacks a membrane-targeting PH domain, it can associate with membranes via its FFAT motif and patches of charged amino acid residues [33]. The current data suggests that it is capable of mediating inter-organelle lipid transport by a' countercurrent' mechanism: the transport of cholesterol to the PM in exchange for PI4,5 $\mathrm{P}_{2}$ [33]. In this respect it thus resembles the 'short' yeast ORPs Osh4p, Osh6 and Osh7p [19,20]. In addition to lipid transport and metabolism, ORP2 also executes functions in cell signaling. This could obviously be secondary to changes in membrane lipid compositions or lateral organization, but our recent findings suggest that protein-protein interactions may play crucial roles here. The role of ORP2 in PI3K/Akt signaling may involve interactions with IQGAP1, Cdc37 and Hsp90, while its functions in F-actin regulation could involve the observed interactions with DIAPH1, SEPT9, ARHGAP12 and MLC12. Moreover, the capacity of ORP2 to bind and transport PIPs is apparently important for its functions in F-actin regulation [35].

To gain a thorough understanding of the physiologic function of ORP2, progress on three major goals is required: (i) The role of lipid binding by ORP2 in terms of the functions of this protein in signaling and F-actin regulation needs to be elucidated. (ii) ORP2/Osbpl2 knockout mice need to be carefully characterized in terms of their cholesterol/lipid and carbohydrate metabolism, insulin signaling, hearing and other F-actin-dependent physiologic processes. (iii) Since ORP2 is expressed ubiquitously in many cell types, its activities need to be investigated in the functional contexts of distinct cell types both in culture and in vivo by employing tissue/cell type-specific knock-out animal models. Additionally, the localization of ORP2 at the ER, LDs and late endocytic compartments raises questions of its possible functions in ER stress responses and lipophagy. Elucidating in detail the functions of ORP2 and other OSBP-related gene products will most certainly open new avenues in the research of organelle lipid homeostasis and their coordination with signaling events that control functions such as cell adhesion, motility and proliferation.

\section{Disclosure}

The authors have no conflicts of interest to disclose concerning this work.

\section{Acknowledgements}

The work in the group is supported by the Adacemy of Finland (grant 285223 to VMO), the Sigrid Juselius Foundation, the Finnish Foundation for Cardiovascular Research, the Magnus Ehrnrooth Foundation, the Liv och Hälsa Foundation, the Novo Nordisk Foundation and the Paavo Nurmi Foundation (VMO).

\section{References}

[1] S. Laitinen, V.M. Olkkonen, C. Ehnholm, E. Ikonen, Family of human oxysterol binding protein (OSBP) homologues. A novel member implicated in brain sterol metabolism, J. Lipid Res. 40 (1999) 2204-2211.

[2] C.J. Jaworski, E. Moreira, A. Li, R. Lee, I.R. Rodriguez, A family of 12 human genes containing oxysterol-binding domains, Genomics 78 (2001) 185-196.

[3] M. Lehto, S. Laitinen, G. Chinetti, M. Johansson, C. Ehnholm, B. Staels, E. Ikonen, V.M. Olkkonen, The OSBP-related protein family in humans, J. Lipid Res. 42 (2001) 1203-1213.

[4] Y. Xu, Y. Liu, N.D. Ridgway, C.R. McMaster, Novel members of the human oxysterol-binding protein family bind phospholipids and regulate vesicle transport, J. Biol. Chem. 276 (2001) 18407-18414.

[5] A.M. Anniss, J. Apostolopoulos, S. Dworkin, L.E. Purton, R.L. Sparrow, An oxysterol-binding protein family identified in the mouse, DNA Cell Biol. 21 (2002) $571-580$

[6] Y.J. Im, S. Raychaudhuri, W.A. Prinz, J.H. Hurley, Structural mechanism for sterol sensing and transport by OSBP-related proteins, Nature 437 (2005) 154-158.

[7] M.K. Manik, H. Yang, J. Tong, Y.J. Im, Structure of yeast OSBP-related protein Osh1 reveals key determinants for lipid transport and protein targeting at the nucleusvacuole Junction, Structure 25 (2017) 617-629 e613.

[8] J. Tong, H. Yang, S.H. Eom, Y.J. Im, Structure of osh3 reveals a conserved mode of phosphoinositide binding in oxysterol-binding proteins, Structure 21 (2013) 1203-1213.

[9] C.J. Loewen, A. Roy, T.P. Levine, A conserved ER targeting motif in three families of lipid binding proteins and in Opilp binds VAP, EMBO J. 22 (2003) 2025-2035.

[10] X. Du, J. Kumar, C. Ferguson, T.A. Schulz, Y.S. Ong, W. Hong, W.A. Prinz, R.G. Parton, A.J. Brown, H. Yang, A role for oxysterol-binding protein-related protein 5 in endosomal cholesterol trafficking, J. Cell Biol. 192 (2011) 121-135.

[11] D. Yan, M.I. Mäyränpää, J. Wong, J. Perttilä, M. Lehto, M. Jauhiainen, P.T. Kovanen, C. Ehnholm, A.J. Brown, V.M. Olkkonen, OSBP-related protein 8 (ORP8) suppresses ABCA1 expression and cholesterol efflux from macrophages, J Biol. Chem. 283 (2008) 332-340.

[12] B. Mesmin, B. Antonny, The counterflow transport of sterols and PI4P, Biochim. Biophys. Acta 1861 (2016) 940-951.

[13] V.M. Olkkonen, S. Li, Oxysterol-binding proteins: sterol and phosphoinositide sensors coordinating transport, signaling and metabolism, Prog. Lipid Res. 52 (2013) 529-538.

[14] A. Pietrangelo, N.D. Ridgway, Bridging the molecular and biological functions of the oxysterol-binding protein family, Cell. Mol. Life Sci. 75 (2018) 3079-3098.

[15] J. Chung, F. Torta, K. Masai, L. Lucast, H. Czapla, L.B. Tanner, P. Narayanaswamy, M.R. Wenk, F. Nakatsu, P. De Camilli, INTRACELLULAR TRANSPORT. PI4P/ phosphatidylserine countertransport at ORP5- and ORP8-mediated ER-plasma membrane contacts, Science 349 (2015) 428-432.

[16] M. de Saint-Jean, V. Delfosse, D. Douguet, G. Chicanne, B. Payrastre, W. Bourguet, B. Antonny, G. Drin, Osh4p exchanges sterols for phosphatidylinositol 4-phosphate between lipid bilayers, J. Cell Biol. 195 (2011) 965-978.

[17] B. Mesmin, J. Bigay, J. Moser von Filseck, S. Lacas-Gervais, G. Drin, B. Antonny, A four-step cycle driven by PI(4)P hydrolysis directs sterol/PI(4)P exchange by the ER-Golgi tether OSBP, Cell 155 (2013) 830-843.

[18] B. Mesmin, J. Bigay, J. Polidori, D. Jamecna, S. Lacas-Gervais, B. Antonny, Sterol transfer, PI4P consumption, and control of membrane lipid order by endogenous OSBP, EMBO J. 36 (2017) 3156-3174.

[19] J. Moser von Filseck, A. Copic, V. Delfosse, S. Vanni, C.L. Jackson, W. Bourguet, G. Drin, INTRACELLULAR TRANSPORT. Phosphatidylserine transport by ORP/Osh 
proteins is driven by phosphatidylinositol 4-phosphate, Science 349 (2015) 432-436.

[20] J. Moser von Filseck, S. Vanni, B. Mesmin, B. Antonny, G. Drin, A phosphatidylinositol-4-phosphate powered exchange mechanism to create a lipid gradient between membranes, Nat. Commun. 6 (2015) 6671.

[21] R. Ghai, X. Du, H. Wang, J. Dong, C. Ferguson, A.J. Brown, R.G. Parton, J.W. Wu, H. Yang, ORP5 and ORP8 bind phosphatidylinositol-4, 5-biphosphate (PtdIns $(4,5) \mathrm{P}$ 2) and regulate its level at the plasma membrane, Nat. Commun. 8 (2017) 757.

[22] B. Antonny, J. Bigay, B. Mesmin, The oxysterol-binding protein cycle: burning off PI (4)P to transport cholesterol, Annu. Rev. Biochem 87 (2018) 809-837.

[23] M. Weber-Boyvat, H. Kentala, J. Peränen, V.M. Olkkonen, Ligand-dependent localization and function of ORP-VAP complexes at membrane contact sites, Cell. Mol. Life Sci. 72 (2015) 1967-1987.

[24] S. Laitinen, M. Lehto, S. Lehtonen, K. Hyvärinen, S. Heino, E. Lehtonen, C. Ehnholm, E. Ikonen, V.M. Olkkonen, ORP2, a homolog of oxysterol binding protein, regulates cellular cholesterol metabolism, J. Lipid Res. 43 (2002) 245-255.

[25] R. Hynynen, M. Suchanek, J. Spandl, N. Back, C. Thiele, V.M. Olkkonen, OSBPrelated protein 2 is a sterol receptor on lipid droplets that regulates the metabolism of neutral lipids, J. Lipid Res. 50 (2009) 1305-1315.

[26] M. Suchanek, R. Hynynen, G. Wohlfahrt, M. Lehto, M. Johansson, H. Saarinen, A. Radzikowska, C. Thiele, V.M. Olkkonen, The mammalian OSBP-related proteins (ORP) bind 25-hydroxycholesterol in an evolutionarily conserved pocket, Biochem. J. 405 (2007) 473-480.

[27] H. Kentala, A. Koponen, H. Vihinen, J. Pirhonen, G. Liebisch, Z. Pataj, A. Kivelä, S. Li, L. Karhinen, E. Jääskeläinen, R. Andrews, L. Meriläinen, S. Matysik, E. Ikonen, Y. Zhou, E. Jokitalo, V.M. Olkkonen, OSBP-related protein-2 (ORP2): a novel Akt effector that controls cellular energy metabolism, Cell. Mol. Life Sci. 75 (2018) 4041-4057.

[28] H. Kentala, S.G. Pfisterer, V.M. Olkkonen, M. Weber-Boyvat, Sterol liganding of OSBP-related proteins (ORPs) regulates the subcellular distribution of ORP-VAPA complexes and their impacts on organelle structure, Steroids 99 (2015) 248-258.

[29] A. Koponen, A. Arora, K. Takahashi, H. Kentala, A. Kivelä, E. Jääskeläinen, J. Peränen, P. Somerharju, E. Ikonen, T. Viitala, V.M. Olkkonen, ORP2 interacts with phosphoinositides and controls the subcellular distribution of cholesterol, Biochimie 158 (2018) 90-101.

[30] M. Thoenes, U. Zimmermann, I. Ebermann, M. Ptok, M.A. Lewis, H. Thiele, S. Morlot, M.M. Hess, A. Gal, T. Eisenberger, C. Bergmann, G. Nurnberg, P. Nurnberg, K.P. Steel, M. Knipper, H.J. Bolz, OSBPL2 encodes a protein of inner and outer hair cell stereocilia and is mutated in autosomal dominant hearing loss (DFNA67), Orphanet J. Rare Dis. 10 (2015) 15.

[31] T. Escajadillo, H. Wang, L. Li, D. Li, M.B. Sewer, Oxysterol-related-binding-protein related protein-2 (ORP2) regulates cortisol biosynthesis and cholesterol homeostasis, Mol. Cell. Endocrinol. 427 (2016) 73-85.

[32] R. Hynynen, S. Laitinen, R. Käkelä, K. Tanhuanpää, S. Lusa, C. Ehnholm, P. Somerharju, E. Ikonen, V.M. Olkkonen, Overexpression of OSBP-related protein 2 (ORP2) induces changes in cellular cholesterol metabolism and enhances endocytosis, Biochem. J 390 (2005) 273-283.
[33] H. Wang, Q. Ma, Y. Qi, J. Dong, X. Du, J. Rae, J. Wang, W.-F. Wu, A.J. Brown, R.G. Parton, J.-W. Wu, H. Yang, ORP2 delivers cholesterol to the plasma membrane in exchange for phosphatidylinositol 4,5-bisphosphate $\left(\mathrm{PI}(4,5) \mathrm{P}_{2}\right)$, Mol. Cell 73 (2019) 1-16.

[34] M. Jansen, Y. Ohsaki, L. Rita Rega, R. Bittman, V.M. Olkkonen, E. Ikonen, Role of ORPs in sterol transport from plasma membrane to ER and lipid droplets in mammalian cells, Traffic 12 (2011) 218-231.

[35] H. Kentala, A. Koponen, A.M. Kivelä, R. Andrews, C. Li, Y. Zhou, V.M. Olkkonen, Analysis of ORP2-knockout hepatocytes uncovers a novel function in actin cytoskeletal regulation, FASEB J. 32 (2018) 1281-1295.

[36] D. Li, E.B. Dammer, N.C. Lucki, M.B. Sewer, cAMP-stimulated phosphorylation of diaphanous 1 regulates protein stability and interaction with binding partners in adrenocortical cells, Mol. Biol. Cell 24 (2013) 848-857.

[37] N. Watanabe, P. Madaule, T. Reid, T. Ishizaki, G. Watanabe, A. Kakizuka, Y. Saito, K. Nakao, B.M. Jockusch, S. Narumiya, p140mDia, a mammalian homolog of Drosophila diaphanous, is a target protein for Rho small GTPase and is a ligand for profilin, EMBO J. 16 (1997) 3044-3056.

[38] R. Käkelä, K. Tanhuanpää, S. Laitinen, P. Somerharju, V.M. Olkkonen, Overexpression of OSBP-related protein 2 (ORP2) in CHO cells induces alterations of phospholipid species composition, Biochem. Cell Biol. 83 (2005) 677-683.

[39] M. Fleischmann, P.B. Iynedjian, Regulation of sterol regulatory-element binding protein 1 gene expression in liver: role of insulin and protein kinase B/cAkt, Biochem. J. 349 (2000) 13-17.

[40] T. Porstmann, B. Griffiths, Y.L. Chung, O. Delpuech, J.R. Griffiths, J. Downward, A. Schulze, PKB/Akt induces transcription of enzymes involved in cholesterol and fatty acid biosynthesis via activation of SREBP, Oncogene 24 (2005) 6465-6481.

[41] J.L. Yecies, H.H. Zhang, S. Menon, S. Liu, D. Yecies, A.I. Lipovsky, C. Gorgun, D.J. Kwiatkowski, G.S. Hotamisligil, C.H. Lee, B.D. Manning, Akt stimulates hepatic SREBP1c and lipogenesis through parallel mTORC1-dependent and independent pathways, Cell Metab. 14 (2011) 21-32.

[42] E. Beurel, S.F. Grieco, R.S. Jope, Glycogen synthase kinase-3 (GSK3): regulation, actions, and diseases, Pharmacol. Ther. 148 (2015) 114-131.

[43] S. Choi, R.A. Anderson, IQGAP1 is a phosphoinositide effector and kinase scaffold, Adv Biol Regul 60 (2016) 29-35.

[44] A.D. Basso, D.B. Solit, G. Chiosis, B. Giri, P. Tsichlis, N. Rosen, Akt forms an intracellular complex with heat shock protein 90 (Hsp90) and Cdc37 and is destabilized by inhibitors of Hsp90 function, J. Biol. Chem. 277 (2002) 39858-39866.

[45] G. Xing, J. Yao, B. Wu, T. Liu, Q. Wei, C. Liu, Y. Lu, Z. Chen, H. Zheng, X. Yang, $\mathrm{X}$. Cao, Identification of OSBPL2 as a novel candidate gene for progressive nonsyndromic hearing loss by whole-exome sequencing, Genet. Med. 17 (2015) $210-218$.

[46] J. McGrath, P. Roy, B.J. Perrin, Stereocilia morphogenesis and maintenance through regulation of actin stability, Semin. Cell Dev. Biol. 65 (2017) 88-95.

[47] T. Ueyama, Y. Ninoyu, S.Y. Nishio, T. Miyoshi, H. Torii, K. Nishimura, K. Sugahara, H. Sakata, D. Thumkeo, H. Sakaguchi, N. Watanabe, S.I. Usami, N. Saito, S.I. Kitajiri, Constitutive activation of DIA1 (DIAPH1) via C-terminal truncation causes human sensorineural hearing loss, EMBO Mol. Med. 8 (2016) 1310-1324. 